\title{
Design of a Novel, Battery-less, Solar Powered Wireless Tag for Enhanced Range Remote Tracking Applications
}

\author{
Rushi J. Vyas", Vasileios Lakafosis, Zissis Konstas, Manos M. Tentzeris \\ School of Electrical \& Computer Engineering, Atlanta, GA 30332, USA \\ *E-mail: Rushi.Vyas@gatech.edu
}

\section{Introduction}

This paper introduces a novel battery-less solar powered wireless tag for remote tracking application. A combination of different technologies, namely Photo-voltaics, basic analog circuit design, embedded systems, RF and Microwave/antenna design are utilized to establish an asynchronous wireless link between the solar powered tag and a commercial wireless (WSN) network mote using a simplified protocol in the absence of a regulated battery supply. The design utilizes super capacitors, which are much cleaner to dispose environmentally and have much higher recharge lifetimes compared to batteries. Specifically, a paper-based microcontroller-enabled wireless sensor prototype has been developed for the first time for the UHF frequency band (centered at 904.4 MHz).

\section{System Level Design}

The fundamental problem with integrating the solar powered tag was the scarce output power available from sunlight. The palm-sized solar cell array was capable of generating a maximum of $15 \mathrm{~mW}$. The most power hungry portion of the tag which was the wireless front-end consumed a peak power of $48 \mathrm{~mW}$ in transmit mode. A comparison of the 2 numbers showed that while it was not possible to continuously power the tag with the solar cell array, in a relatively short period of time enough solar energy could be harnessed from the environment to supply the tag with just enough power for communication for a short period of time. Such discontinuity is acceptable for RFIDs, which use asynchronous communication. A system level diagram of the tag is shown in Fig.1. The power source of the tag comprised of an array of Solar Cells configured to give out an open circuit voltage and current above a certain minimum value under different light conditions. The Solar Cell array (PV cells) was interfaced to the tag through a Power Management Circuit (PMU), which was in turn interfaced to the integrated 8-bit microcontroller unit (MCU) housed in the same chip as the Wireless front-end. The MCU was programmed to work with the PMU in monitoring the harnessed power levels and accordingly trigger the wireless communication by implementing the communication protocol and physically controlling the wireless front end of the system. The wireless front-end comprised of a Phase Locked Loop (PLL) with a single-ended Power Amp (PA) at its output at which an appropriately designed external printed monopole antenna was connected.

\section{Solar Cell Array}

The Solar cells were arranged in an array of 5 by 4 big enough to fit in a palm sized circuit board while at the same time be able to harness enough solar power. Each solar cell unit was a stack-up of GaAs cells with open circuit voltage and short circuit current characteristics with respect to light intensity shown in Fig. 2 [1]. Lux Measurements in Atlanta show light intensity to vary from 4,000 lux on a cloudy day to 60,000 lux on a bright clear sunny day. From Fig 2, the open circuit voltage and short circuit current of 
each solar cell of the PV array would vary from between 7 to 10 volts and 0-80 uA based on external light conditions. The parallel configuration of the solar cells would give a maximum short circuit output current of $1.6 \mathrm{~mA}$ [2] while ensuring the same output voltage. In the absence of batteries, the solar energy was to be collected in a capacitor (charge tank) for use by the tag. A higher solar cell output voltage across the capacitor would provide a faster charge-up time for different light conditions, which is important for more frequent communication by the tag as will be shown later.

\section{Power Management Unit (PMU)}

The Power management unit served as the interface between the solar cells and the tag. It was comprised of a large capacitor that served as the solar energy storage device and discrete level circuitry that worked in conjunction with the MCU in the tag as a charge detector. The PMU would keep the tag in either "off" or "sleep" mode till enough of the solar energy had been collected across the charge tank capacitor represented by the capacitor voltage. Once the capacitor voltage had reached a certain user set threshold $\left(\mathrm{V}_{\mathrm{TH}}\right)$, the PMU would trigger the tag on. Once "on", the MCU of the tag would take over control of the operation of the tag. The MCU firmware was designed to optimize power consumption of the tag and the MCU code was implemented to carry out data processing of the wireless data bits in as few instructions as possible to conserve power. In addition, the MCU was also programmed to power on the wireless front-end only when the tag was ready to transmit data. This was done keeping in mind that the power consumption of the MCU at around $500 \mathrm{uA}$ was only a fraction of that consumed by wireless front end at around $15.5 \mathrm{~mA}$. Upon completion of communication, the MCU would discharge the charge tank capacitor to the turn off voltage of the tag through a voltage controlled MOSFET switch effectively resetting the tag. At this point the capacitor would start charging again from the Solar cell array and repeat the process. With this method, the tag turned on only at a certain voltage threshold ensured that the wireless output power of the PA out through the antenna remained the same during each transmits independent of external light conditions. This is important in order to ensure that the received signal strength RSSI $\mathbf{n}$ the receiver side did not change with respect to light and ensure proper triangulation of the received signals for all light conditions. The charge tank capacitance value determined the total transmit time and along with the external light conditions also governed the time interval between adjacent transmits. The minimum lenth of the transmit time is is set by the length of data that needs to be transmitted per energy duty cycle. For the end application of this tag 3 transmits of a data were required to be transmitted per energy duty cycle, for which at $20 \mathrm{kBaud}$ a charge tank capacitance of $637 \mathrm{uF}$ was used. This yielded a total transmit time of close to 43 usec for an FSK modulated signal shown in fig 3 below. Measured transmit time intervals for the chosen charge tank capacitance for light intensities between 10,500 and 70,000 lux represented by the voltage across the charge tank capacitors is shown in fig. 4 below. External light conditions in the lab were replicated using halogen bulbs and wireless measurements were carried out with a Tektronics RSA-3408A real time spectrum analyzer.

\section{Wireless Communication of RFID with WSN}

The receiver for the remote tracking application comprised of a series of commercial Crossbow's MICA2 WSN mote placed around the solar powered tag. Communication between the tag and motes was implemented using a specifically designed communication protocol that was programmed, tried and tested with all the devices in the field. The protocol was optimized to ensure that communication occurred between them 
inspite of effects such as fading, multipath scattering \& bit errors present in outdoor environments, and the limited amount of power harnessed that was available per energy duty cycle. The format of the packet sent from the tag and received by the TI CC1000 transceivers present in the Motes consists of the following fields: Preamble, Sync, ADDR, TYPE, Group, Length and CRC, as captured by the RTSA shown in Fig.5. The first two fields are used for the synchronization of the receiver's clock and the latter field, Cyclic Redundancy Check, helps eliminate bit errors occurring within the sent bit sequence by successfully recognizing a corrupted packet and discarding it

\section{Power Amplifier (PA) Characterization and Antenna Design}

The range of the solar powered tag to the MICA2 receiver is directly proportional to the amount of power transferred from the power amplifier (PA) to the transmitter front end to the antenna. Any impedance mismatch between the two can lead to the internal reflection of a part of the power intended to radiate out of the antenna, thereby minimizing range. Instead of designing the antenna to a 50 match, it was decided to design it to the optimum impedance looking out of the amplifier in the transmitter to eliminate a matching network. To determine the optimum load impedance looking out of the PA in the wireless front-end, a load pull analysis was performed on it at $904.4 \mathrm{MHz}$. The optimum load impedance looking out of the PA at close to the transmit frequency of 904.4 MHz after accounting for bias circuit effects was determined to be $36.95-\mathrm{j} 71.77$ • [4]. For this solar powered tag a newly designed printed monopole structure was used that had a wide input bandwidth and an omni-directional radiation pattern, which is important for triangulation in order to ensure that EM fields are radiated out in the direction of all WSNs with the same starting power. The proposed structure for the antenna design was fabricated on paper using inkjet printed technology in-house and is shown as part of the working prototype in Fig 6. The fabricated antenna was found to have a measured input impedance of $37.3-\mathrm{j} 65.96$, which was very close to the optimal impedance looking out of the PA resulting in a very high return loss of less than $-10 \mathrm{~dB}$ over a bandwidth of almost $0.1 \mathrm{GHz}$ around the center frequency. The measured \& simulated return loss, measured range $\&$ radiation pattern of the antenna structured are shown in figs 7,8 and 9 respectively

\section{Conclusion}

In this paper, we present the first ever wireless "Battery-less Solar Powered Wireless Tag" that can also communicate with wireless sensor networks for the first time reported. This RFID is based on the hardware/software codesign of five different technologies, and could set the foundation of ubiquitous ultra-low-cost RFID networks and the first generation of "green" RF electronics offering truly cognitive intelligence.

\section{References}

[1] Clare, "CPC 1832 Solar Cell," Data Sheet, 2008.

[2] Green, Solar Cells: Operating Principles, Technology and Systems Applications, Bridge printery Pty. Ltd., 1992

[3] Texas Instruments "CC 1000", Data Sheet, 2008.

[4] R. Vyas, A. Rida, L. Yang, M.M Tentzeris, "Design, Integration and Characterization of a Novel Paper Based Wireless Sensor Module", IEEE International Microwave Symposium, accepted June, 2008 

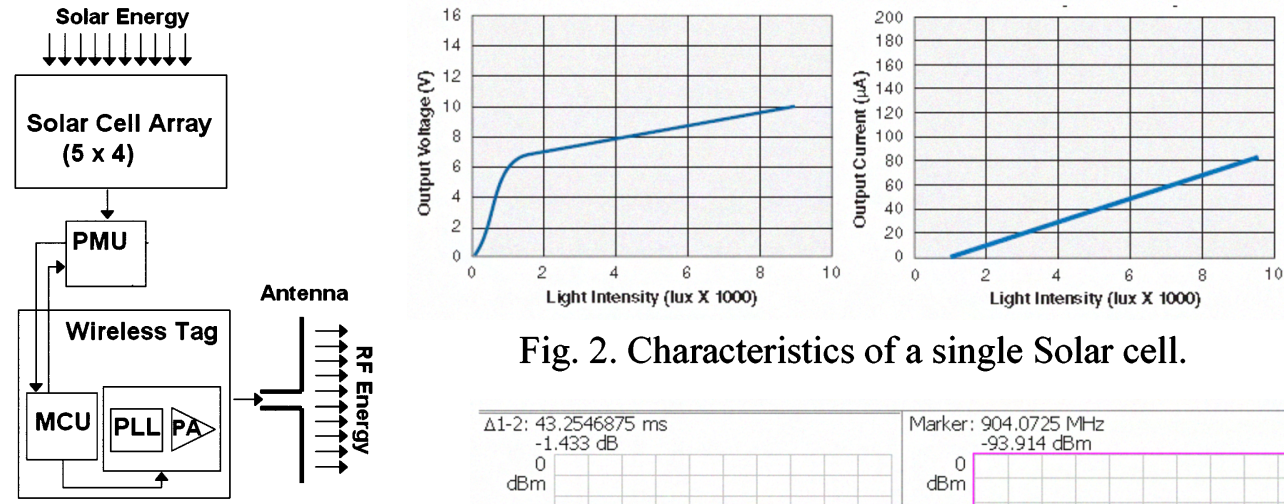

Fig. 2. Characteristics of a single Solar cell.

Fig. 1. Solar Powered Tag System
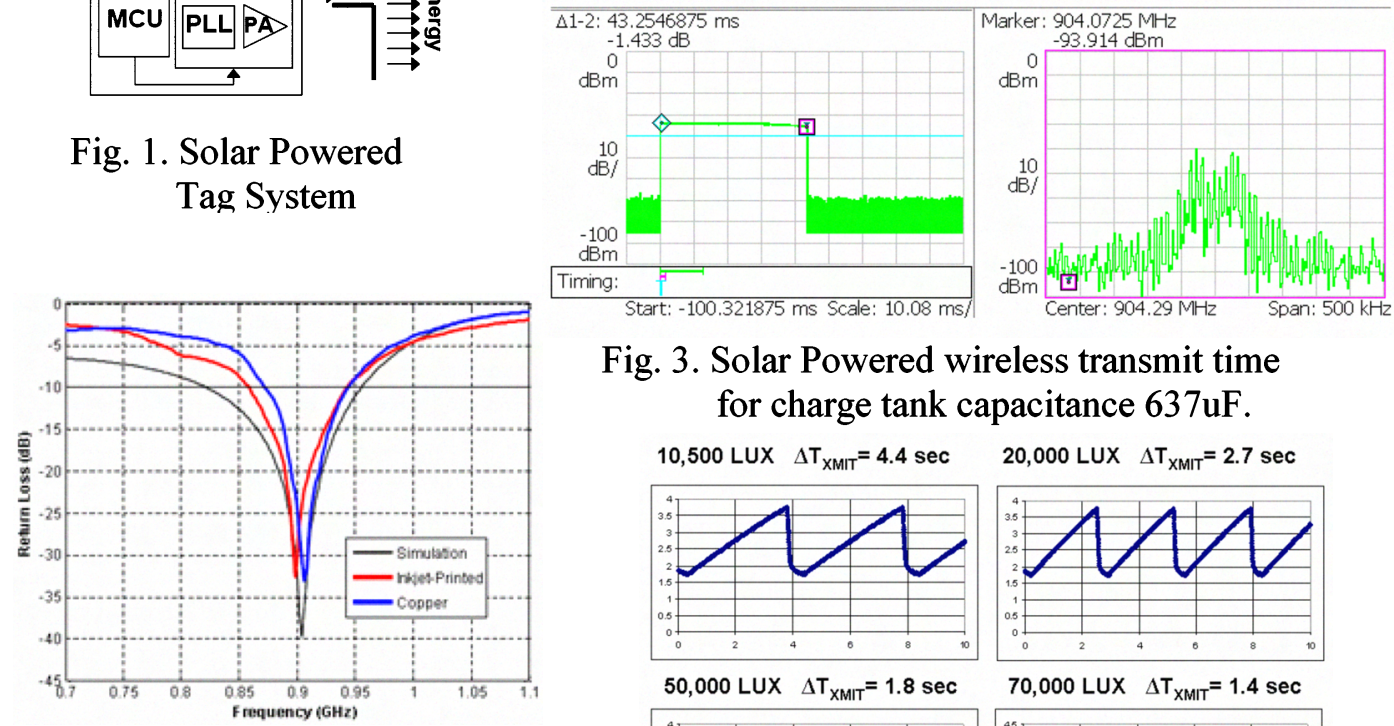

Fig. 3. Solar Powered wireless transmit time for charge tank capacitance $637 \mathrm{uF}$.

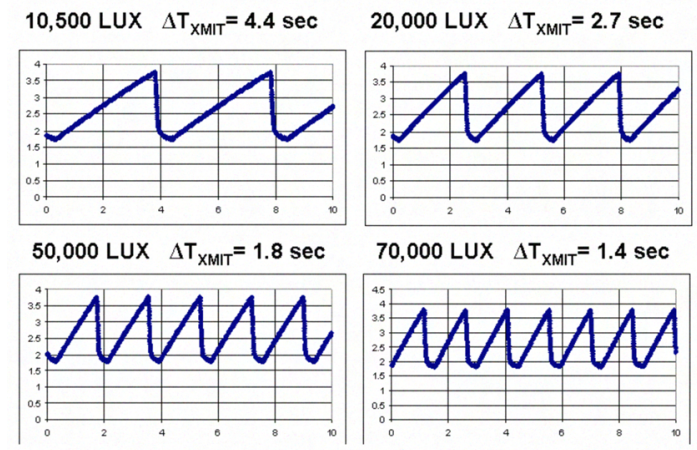

Fig. 4. Transmit Time interval measurements for light intensitites from 10.5 to $70 \mathrm{kLux}$.

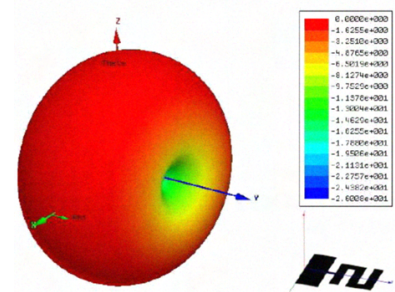
Sunlight $\sim 30 \mathrm{kLux}$

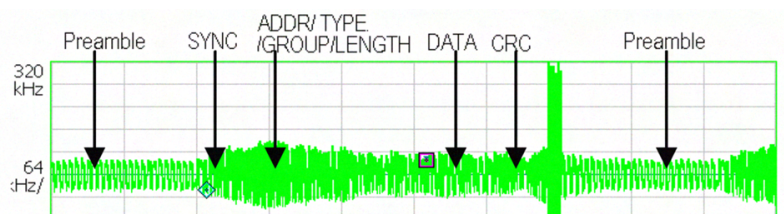

Fig 8. Simulated Antenna radiation pattern

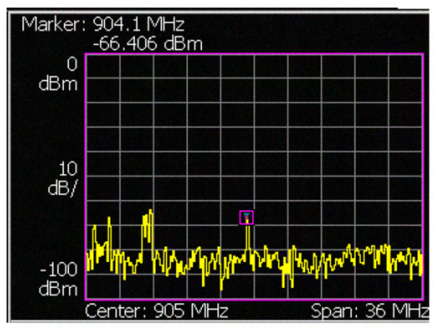

Fig 9. Measured Tag Range. $\sim 500 \mathrm{ft}$. rig. J. solar Powered Wireless Data sequence captured by RTSA

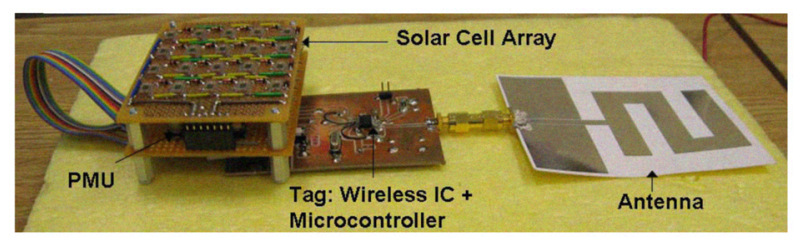

Fig 6. Working Prototype of Solar Powered battery-less Tag. 